\title{
Comparative Evaluation of Pharmacological and Surgical Pregnancy Termination Methods (Magadan region example)
}

\author{
Yaroslav N. Pavlov ${ }^{1, *}$, Nadezhda V. Savvina ${ }^{1}$ and Irina L. Savvina ${ }^{1}$ \\ ${ }^{I}$ M.K. Ammosov North-Eastern Federal University, Department of Public Health and Health, General Hygiene and \\ Bioethics, Yakutsk, 677000, Sakha Yakutia Republic, Russia \\ ${ }^{*}$ Corresponding author. Email: pyn5552007@yandex.ru
}

\begin{abstract}
The problem of artificial abortions of pregnancy, especially in the first trimester, is extremely promising in modern medical practice. The relevance of the issue of abortions is the risk of fatal complications. The purpose was to a comparative assessment of the results of medicinal and operational abortion in the first trimester. The examination of 200 respondents who subjected medical termination in the early stages of pregnancy was performed. They were divided into the first group $(\mathrm{n}=100)$ - a medicinal method and the second $(\mathrm{n}=100)$ - with surgical therapy. $(25.1 \pm 5.63$ years). Patients of the first group used Mifepristone and Misoprostol. Women of the second group were assigned to vacuum aspiration. The study included three stages: clinical, psychological, and pharmacoeconomical. The level of complications that develop after abortion, depending on the method used (medicinal and surgical), does not significantly differ. The endometrial thickness from 1 to $4 \mathrm{~mm}$, detected during 7-10 days after the termination, was more in patient with concretive abortion of pregnancy. The level of vaginal microflora, analyzed on the 13-14 day of abortion, did not have a significant correlation with the type of method of termination of pregnancy (medical and surgical). Artificial abortion in the early stages of pregnancy is accompanied by the formation of psychoemotional both before and after the operation. The pharmacoeconomical cost of medicinal abortion significantly exceeds the operational method. Consequently, the study indicators confirmed one important fact that the values of the studied parameters of clinical examinations, in particular psychoemotional, and economic analyses on the background of the use of pharmacological abortion are close to the indicators of surgical termination.
\end{abstract}

Keywords: pregnancy termination methods, artificial abortions, medical termination, vacuum aspiration, Magadan region

\section{INTRODUCTION}

Artificial abortion plays an important role in organizing reproductive potential, including the unity of gynaecological, somatic, mental health and social factors, taking into account the impact on the realization of reproductive function in the Russian Federation [1].

Despite the annual decrease over the past five years as a parameter of abortion, their number in Russia exaggerates it in all developed countries by about three times. In Italy, there are 20 abortions per 100 births, in
Spain - 10, in Sweden - 40, in the Netherlands -5 , in the UK - $30[2,3]$.

According to researches, after repeated abortion, serious complications were observed (reproductive 21.9-30.2 \%, hyperestrogeny - 3.9-6.2\%, metabolic $3.4-4.8 \%$, uterine perforation $-1.5-4.2 \%$ ). The low level of progesterone secretion in blood plasma was accompanied by the development of proliferative disorders in the reproductive system and manifests itself in the form of the development of uterine neoplasm (endometriosis, myoma, hyperplasia, polyps, etc.), polycystic ovaries [4]. 
Artificial abortion, carried out in early stage of pregnancy, is also characterized by formation of mental and emotional disorders manifesting in somatovegetative changes and nerve-reflex excitability [5].

Nowadays special attention is paid to finding alternative (surgical therapy) methods of abortion in the first trimester of pregnancy by conservative way and antiprogestins [6].

Surgical method (vacuum - aspiration of gestational sac "mini-abortion") which was effective for extracting uterine contents can also be used for abortion in early pregnancy $[7,8]$.

Pharmacological termination of pregnancy excludes injuries of uterine structural layers, but is accompanied by probability of functional changes [9].

According to the World Health Organization, the reliability of induced abortion, the reduction of the maternal mortality parameter and the possibility of forming post-abortion fatal complications depend on the spectrum of conditions - the level and scale of clinical care, the degree of legalization of the abortion method, the use of reliable therapeutic regimens, the introduction of early preventive measures to reduce the risk of complications and rapid rehabilitation of patients after abortion [10].

So, the artificial termination of pregnancy seems to be a general medical issue, along with this, the selection of this method in remains controversial and little studied.

The purpose of the research is a comparative study of the results of pharmacological and surgical abortion in the first trimester of pregnancy.

\section{MATERIALS AND METHODS}

200 patients who underwent an abortion in the early stages of pregnancy at Magadan Maternity Hospital were examined.

Criteria for including patients in this research were written individual consent of patients to participate in this study; clinical, biochemical and instrumental verification of pregnancy diagnosis; pregnancy less than 12 weeks; from 19 to 49 years age of the patients being examined; mild comorbidities.

Criteria for excluding patients from this research: personal refusal of a patient to participate in the study; violation of the report of the study or medical instruction; over 49 years of age and under 19 years of age; pregnancy longer than 12 weeks; severe comorbidities (somatic, psychological, oncological).

Patients were divided depending on the method of abortion into 2 groups: the first (main, $\mathrm{n}=100$ ) - the drug method was used (age was $24.8 \pm 4.25$ years) and the second (comparison, $\mathrm{n}=100$ ) - with surgical therapy $(25.1 \pm 5.63$ years $)$.

Artificial abortion of the first group was performed with Mifepristone (Mifepristone, G03XB01, No. 002340/01, $600 \mathrm{mg}$ ) and Misoprostol (Mizoprostol, A02BB01, No. LS - 001766, $400 \mu \mathrm{g}$ ). These drugs are allowed for use in medicine in accordance with the law of the Russian Federation of November 21, 2011. No. 323 - Federal Law.

Women of the second group were assigned a vacuum - aspiration of gestational sac with a cannula (4-7 mm). Surgery was performed subject to aseptic and antiseptic rules. Pain relief - individual: topically with novocaine $0.25 \%$, intravenously - ketamine $(0.5-4.5 \mathrm{mg} / \mathrm{kg})$.

According to the clinical characteristic, it was found that the term of abortion in patients of the first group was $4.9 \pm 0.03$ week ( $p>0.05)$, in the second group $5.5 \pm 0.05$ week $(\mathrm{p}>0.05)$

The average duration of the onset of the first periods in women of the main group was $13.01 \pm 0.98$ years old In the comparison group, this indicator was equal $13.8 \pm 1.09$ years.

Sexual life in the studied women with pharmacological abortion occurred at $18.9 \pm 4.27$ years and $19.4 \pm 2.87$ years in patients with a surgical method of termination of pregnancy.

The study of reproductive behavior found out that 45 women of the first group $(45.0 \%)$ and 47 women of the second group $(47.0 \%)$ were first pregnancy. The percentage of use of different methods of contraception in the main group was $51.0 \%$, and in the comparison group $-47.0 \%(p>0.05)$. However, the proportion of use of modern contraception in the studied patients was low: $9(9.0 \%)$ in the first group and $11(11.0 \%)$ in the second $(\mathrm{p}>0.05)$.

The gynecological pathologies (chronic extragenital) was established in 35 respondents of the main group $(35.0 \%)$ and $39(39.0 \%)$ - in the second group.

The level of education were higher and secondary education, the frequency of which was $48(48.0 \%)$ and $52(52.0 \%)$ in the group with a drug-induced abortion method and $51(51.0 \%)$ and $49(49.0 \%)$ in the group with operative method.

The number of children in the study groups was different. In the main group, one child was in 40 $(40.0 \%)$, and in the second group $-42(42.0 \%)$. Two and three children $-10(10.0 \%)$ and $7(7.0 \%)$ and 5 $(5.0 \%)$ and $4(4.0 \%)$, respectively.

When studying marital status, it was noted that 43 respondents of the first group $(43.0 \%)$ and 41 women of the second group $(41.0 \%)$ had a registered marriage, the actual $-50(50.0 \%)$ and $52(52.0 \%)$, 
respectively, and no partner was in $8(8.0 \%)$ and 7 $(7.0 \%)$, respectively.

Consent to abortion decision between the partners was mutual (with the husband) in 80 patients $(80.0 \%$ ) of the main group and $78(78.0 \%)$ of the comparison group.

The stages of this work are three within 14 days after the abortion.

1. The first stage is clinical evaluation of the examined patients after abortion. He determined subjective complaints, the duration of blood discharge, culture of the vaginal secretions, complications, an echographic study.

2. The second is the taxing of psychological status before and after abortion with anxiety and depression scale (The hospital Animation and Depression).

3. Third-economic analysis of the methods used.

The obtained digital results were processed by routine methods of statistics - Microsoft Word and Microsoft Excel XP (2013).

\section{RESULTS OF THE RESEARCH}

Clinical manifestations (the first stage of the study) established that the duration of blood discharge in women of the main group (on the background of pharmacological abortion) was $8.2 \pm 0.09$ days and $6.8 \pm 0.04$ days $(\mathrm{p}<0.05)-$ a comparison group (with a surgical abortion) (Fig. 1 (A)).

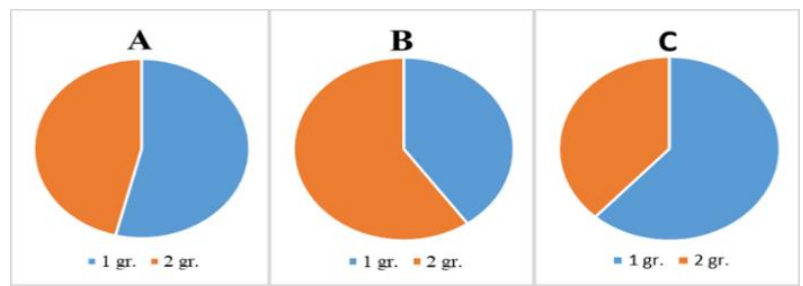

Figure 1. Clinical manifestations. A. duration of vaginal discharge (days). B. Duration of vaginal discharge after abortion (\%). C. Duration of discharges from 6 to 9 days (\%)

The duration of vaginal discharge from 1 to 5 days after abortion in the group with pharmacological method was established in $42(42.0 \%)$, and in 64 (64.0\%) in the group with surgical method of termination (Fig. 1 (B)).

Duration of blood discharge in the period from 6 to 9 days was detected in $58(58.0 \%)$ patients of the first group and in $36(36.0 \%)$ in the second group (Fig. 1 (C)).

So, post-abortion discharges continue longer in women of the main group (where a drug-induced method of termination of pregnancy was taken). A comparative group (on the background of using vacuum - aspiration), had probably the consequence of side effects of antiprogestin.

When assessing the risk of post-abortion complications, we noted that the value of this parameter in the examined groups did not significantly differ. Hematometer development in the uterine cavity, polyps, bleeding and gestational sac residue in the first group were $6(6.0 \%), 7(7.0 \%), 10(10.0 \%)$ and $7(7.0 \%)$, and in the comparison group were $8(8.0 \%), 5(5.0 \%)$, $12(12.0 \%)$ and $4(4.0 \%)$, respectively (Fig. 2).

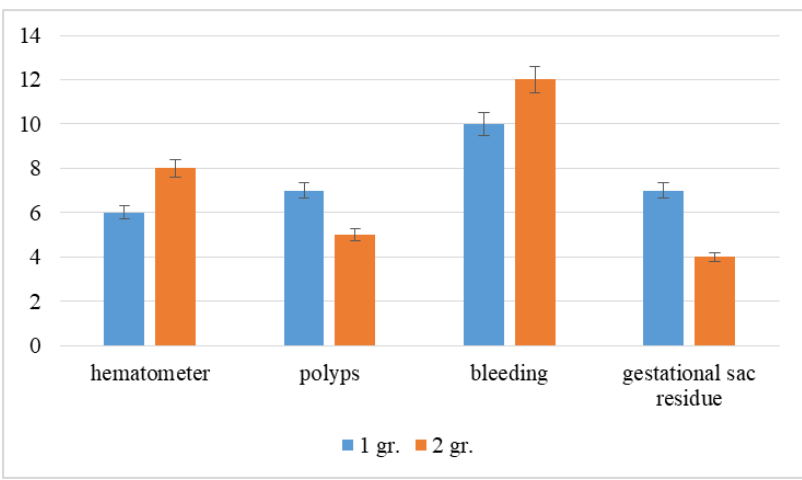

Figure 2. Presence of post-abortion complications (\%)

Conservative therapy, which included antispasmodic, antibiotic, infusion, etc. treatment of the above complications, were prescribed to the patients of the study.

In case of taxation of endometrioid uterine tissue thickness with ultrasound examination in post-abortion period on day $6-9$, determined in $72(72.0 \%)$ patients of the main group (on the background of pharmacological method of termination) and 74 $(74.0 \%)$ women in comparison group (using operative method). At the same time, the average thickness in women of the first group was $7.0 \pm 0.09 \mathrm{~mm}$, and in the second group $-6.1 \pm 0.05 \mathrm{~mm}$. There were no reliable differences between groups for this indicator.

However, statistically significant differences were established among respondents of each group by endometrial thickness from 1 to $4 \mathrm{~mm}$ and from 5 to $8 \mathrm{~mm}$, which in the main group (the drug-induced method of abortion) were $22(22.0 \%)$ and $43(43.0 \%)$, and in the surgical group - $10(10.0 \%)$ and $61(61.0 \%)$ patients.

The excess proportion of patients with endometrial thickness of 1 to $4 \mathrm{~mm}$ in the first group indicates the residual effect of antiprogestin.

Analysis of bacteriological culture of the postabortion vaginal secretions showed minor changes in vaginal microflora in both groups. Before the abortion, mushrooms of the genus Candida were found in 12 $(12.0 \%)$ women of the first group and $9(9.0 \%)$ women 
of the second group. After abortion, the level of these fungi decreased to the normal version: in the main group $-3(3.0 \%)$, and in the comparison group $-2(2.0 \%)$.

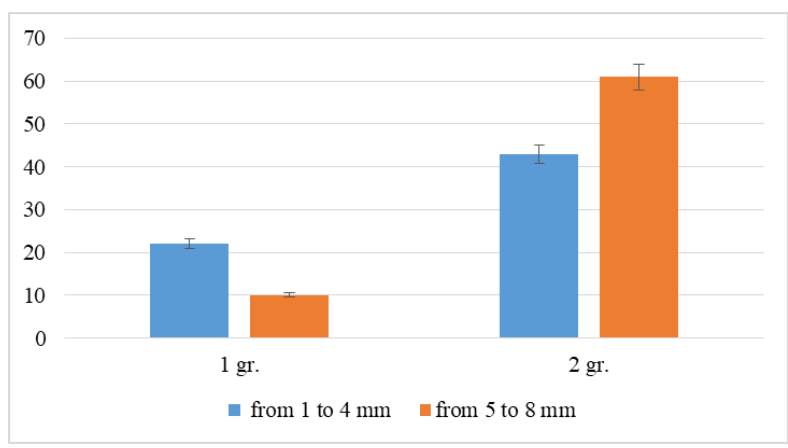

Figure 3. Endometrial thickness (\%)

The microbiological test of the vaginal secretions after pregnancy termination showed the presence of a small number of microorganisms (Escherichia coli, Enterobacter spp., Klebsiella spp., P. aeruginosa), which accounted for $5,3,4$ and $2 \%$ of the respondents of the main group and 8, 6, 5 and $4 \%$ of the women of the comparison group. There was no significant change in the vaginal microflora of the women under study after abortion when using the drug and operative method.

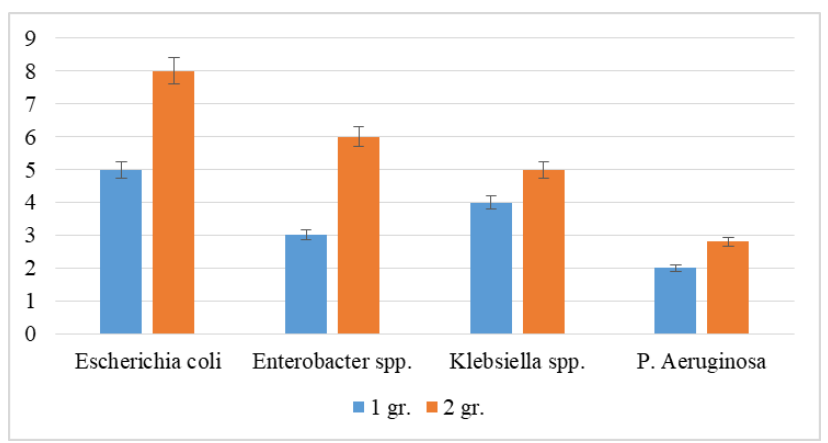

Figure 4. Presence of microorganisms (\%)

A psychological analysis study (second stage) showed the reason and conditions for the choice of the method of pregnancy termination by the examined women: medicinal or surgical.

The main reasons for the choice of drug abortion in patients of the first group were the avoidance of complications (operative, psychoemotional, infectious) $(47.0 \%)$, safety $(30.0 \%)$, high effectiveness $(15.0 \%)$, satisfaction $(8.0 \%$ ) (Fig. 5 (A)). Respondents of the second group chose surgical termination of pregnancy according to the following criteria: reliability $(49.0 \%)$, short completion date $(20.0 \%)$, price availability $(18.0 \%)$, rapid recovery $(13.0 \%)$ (Fig. $5(\mathrm{~B}))$.

When analyzing the psychological impact of using different methods of pregnancy termination in a period of less than 12 weeks, we found out that waiting for an artificial abortion is directly related to anxiety disorders, especially significant when waiting for the use of the pharmacological method of pregnancy termination.
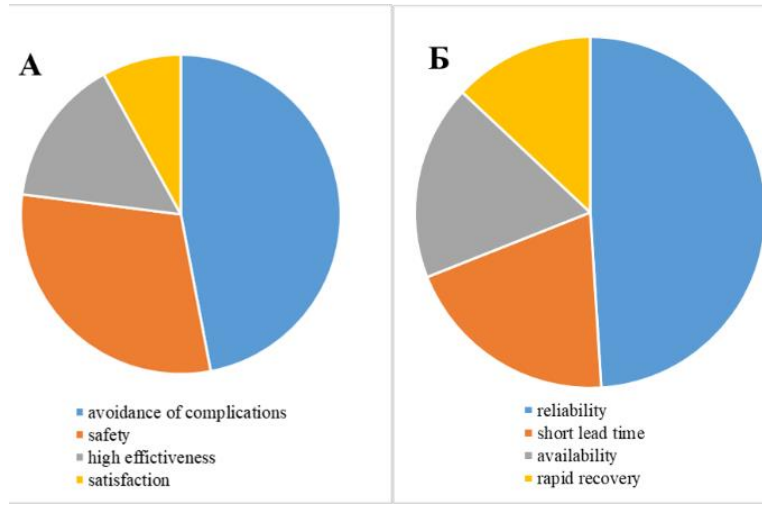

Figure 5. Reasons for choosing abortion method. A. The first group, B. Second group (\%)

Before performing abortion, signs of anxiety were recorded in the main group of $16(16.0 \%)$ patients, and the main group of $43(43.0 \%)$. In the postoperative period, anxiety severity was detected in $21(21.0 \%)$ respondents of the second group and in $51(51.0 \%)$ patients of the comparison group (Fig. 5).

The development of depressive disorders in preoperative time was noted in both women of the main group $(24.0 \%)$ and patients of the comparison group $(19.0 \%)$. Post-abortion follow-up showed signs of depression in $30(30.0 \%)$ women of the first group (where the medication was taken), and in 48 (48.0\%) respondents of the second group (with surgical abortion) (Fig. 6).

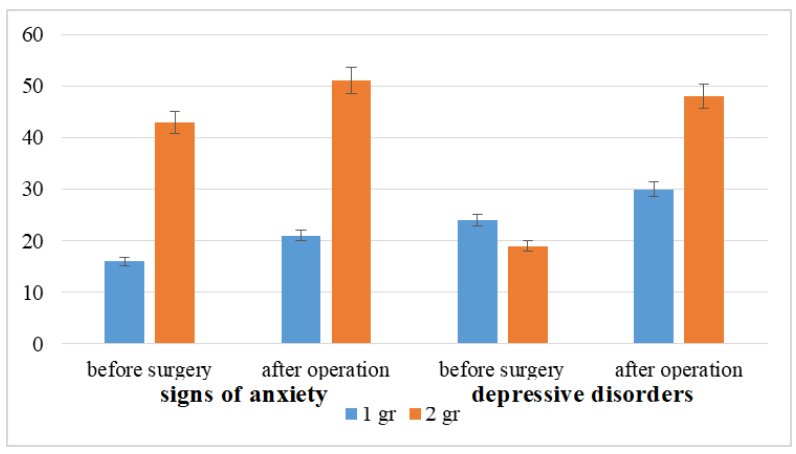

Figure 6. Reasons for choosing abortion method. A. The first group, B. Second group (\%)

Thus, there are reliable differences in the severity of anxiety and depressive disorders in patients on the background of pharmacological abortion in comparison with women prescribed the operative method: 62.7 and $27.0 \%(\mathrm{p}<0.05)$ before performing the abortion and 58.9 and $37.5(\mathrm{p}<0.05)$ after the procedure.

During the economic test (third stage), it was established that the cost of medical abortion amounted 
to 12709.60 thousand rubles per patient and $1,270.960$ million rubles for all respondents of the main group.

During the economic analysis, it is shown that the price of surgical termination (uncomplicated) pregnancy is 13253.00 rubles per woman and 1.325.300 million rubles for all patients of the comparison group.

Thus, the economic difference between drug abortion used in the main group and the surgical method used in the comparison group is 543.4 rubles per patient in the absence of complications and 45.340 thousand rubles for all respondents.

\section{CONCLUSION}

1. Pregnancy termination in the first trimester by the operative method (vacuum - aspiration of the gestational sac) is accompanied by a shorter duration of post-abortion bloody secretions compared to pharmacological abortion.

2. The level of complications developing after abortion, depending on the method used (drug and surgical), does not significantly differ.

3. Endometrial thickness from 1 to $4 \mathrm{~mm}$, diagnosed 7-10 days after abortion, was larger in women with medicinal termination of pregnancy.

4. The level of vaginal microflora, analyzed on the 13-14 day of abortion, did not have a reliable conjugation with the type of method of termination of pregnancy (drug and surgical).

5. Artificial abortion in early pregnancy is accompanied by the development of psychoemotional disorders both in the preoperative period and postoperative.

6. Pharmacoeconomic cost of medicinal abortion significantly exceeds operative method.

\section{REFERENCES}

[1] A.I. Malyshkina, N.V. Batrak, L.P. Peretyatko, M.L. Dobrynina, Clinical-laboratory and morphological differences of undeveloped pregnancy and spontaneous abortion in early pregnancy, Rus. Bull. of obstetrician-gynecologist 21(1) (2021) 31-37.

[2] S.V. Kopan, M.V. Andreeva, K.Yu. Tikhaeva, E.P. Shevtsova, Student view of modern contraception, Bull. of the Volga State Med. Univ. 1(77) (2021).

[3] ESHRE Capri Workshop Group, Induced abortion, Hum. Reprod. 32(6) (2017) 1160-1169. DOI: 10.1093/humrep/dex071

[4] Z.S. Rumyantseva, E.Yu. Lumanova, N.I., Volotskaya, S.S. Anikin, Predictors of undeveloped pregnancy and the role of pregravidar training in the prevention of repeated reproductive loss, Vyatka Med. Bull. 1(69) (2021).

[5] V.A. Smirnov, V.V. Gruzdev, Family policy of the Russian state in great challenges: Main problems and contradictions, ARS Administrandi 13(1) (2021) 71-88.

[6] Y.N. Pavlov, Analysis of the prevalence of abortion and ways to solve the problem in the Magadan region, Far Eastern Med. J. 1 (2021) 8489.

[7] J. Conti, E.P. Cahill, Self-managed abortion, Curr. Opin. Obstet. Gynecol. 31(6) (2019) 435-440. DOI: $10.1097 /$ GCO.0000000000000585

[8] S.G. Vorsanova, Yu.B. Yurov, I.A. Demidova et al., Interphasic molecular and cytogenetic diagnostics of chromosomal anomalies (fish) in material of spontaneous abortions of the first trimester of pregnancy: analysis of 1333 cases, Modern probl. of sci. and ed. 2 (2021) 150.

[9] Y.O. Turbina, N.V. Polunina, Analysis of the prevalence and causes of abortion in the metropolis, Cardiovasc. therapy and prevent. 20(S1) (2021) 85.

[10] I.N. Vorobtsova, M.V. Konovalova, V.V. Vasiliev, L.V. Kurdynko, Pathogenetic features of the downward spread of persistent chlamydia infection after medical abortion, Int. J. of Med. and Psychol. 3(1) (2020) 122-126. 
[11] [9] A. Pnueli, In transition from global to modular temporal reasoning about programs, in: K.R. Apt (Ed.), Logics and Models of Concurrent Systems, Springer, Berlin, Heidelberg, 1984, pp. 123-144. DOI: https://doi.org/10.1007/978-3-642-82453-1_5

[12] [10] B. Meyer, Applying "Design by Contract", Computer 25(10) (1992) 40-51. DOI: https://doi.org/10.1109/2.161279

[13] [11] S. Bensalem, M. Bogza, A. Legay, T.H. Nguyen, J. Sifakis, R. Yan, Incremental component-based construction and verification using invariants, in: Proceedings of the Conference on Formal Methods in Computer Aided Design (FMCAD), IEEE Press, Piscataway, NJ, 2010, pp. 257-256.

[14] [12] H. Barringer, C.S. Pasareanu, D. Giannakopolou, Proof rules for automated compositional verification through learning, in Proc. of the 2nd International Workshop on Specification and Verification of Component Based Systems, 2003.

[15] [13] M.G. Bobaru, C.S. Pasareanu, D. Giannakopoulou, Automated assume-guarantee reasoning by abstraction refinement, in: A. Gupta, S. Malik (Eds.), Proceedings of the Computer Aided Verification, Springer, Berlin, Heidelberg, 2008, pp. 135-148. DOI: https://doi.org/10.1007/978-3-540-70545-1_14 